\title{
O uso de cigarros eletrônicos no Brasil: uma revisão integrativa
}

\author{
The use of electronic cigarettes in Brazil: an integrative review \\ El uso de cigarrillos electrónicos en Brasil: una revisión integradora
}

Recebido: 22/11/2021 | Revisado: 29/11/2021 | Aceito: 30/11/2021 | Publicado: 01/12/2021

\author{
Adeilson Pereira da Silva \\ ORCID: https://orcid.org/0000-0002-6985-531X \\ Universidade Estadual da Paraíba, Brasil \\ E-mail: adeilsonpereira9821@gmail.com \\ Clésia Oliveira Pachú \\ ORCID: https://orcid.org/0000-0002-7356-6297 \\ Universidade Estadual da Paraíba, Brasil \\ E-mail: clesiapachu@hotmail.com
}

\begin{abstract}
Resumo
O cigarro eletrônico foi introduzido no mercado como uma possível terapêutica na cessação do tabagismo. Todavia, os cigarros eletrônicos podem conter diversas substâncias químicas, como nicotina, além de metais pesados, como ferro e chumbo. O presente estudo objetivou analisar o uso de cigarros eletrônicos no Brasil, por meio de uma revisão integrativa. Utilizou-se de revisão integrativa com busca de dados realizada nas bases Scielo, BVS e Google Acadêmico, utilizando os seguintes descritores: "uso de cigarros eletrônicos" e "vaping", realizada no período de setembro a outubro de 2021. Observou-se que são escassos os estudos realizados no Brasil acerca de cigarros eletrônicos. Constatou-se que os cigarros eletrônicos são uma grande tendência, sobretudo entre os jovens do sexo masculino. Podese verificar que o uso de cigarros eletrônicos tem relação com o uso de cigarros convencionais e outras drogas. Ademais, diversos indivíduos acreditam que os cigarros eletrônicos não fazem mal à saúde ou são menos nocivos que os cigarros convencionais. A sensibilização da população acerca dos riscos do uso de cigarros eletrônicos se torna imprescindível no processo educativo, visando alertar os malefícios que estes dispositivos eletrônicos podem ocasionar. Portanto, diante da escassez de publicações científicas acerca do uso de cigarros eletrônicos no Brasil, sugere-se a realização de mais estudos que contemplem a problemática em discussão.
\end{abstract}

Palavras-chave: Cigarro eletrônico; Dispositivo eletrônico; Tabagismo.

\begin{abstract}
Electronic cigarettes were introduced to the market as a possible therapy for smoking cessation. However, e-cigarettes can contain several chemical substances, such as nicotine, in addition to heavy metals, such as iron and lead. This study aimed to analyze the use of electronic cigarettes in Brazil, through an integrative review. An integrative review was used with data search carried out in the Scielo, BVS and Academic Google databases, using the following descriptors: "use of electronic cigarettes" and "vaping", carried out from September to October 2021. It was observed that there are few studies carried out in Brazil on electronic cigarettes. It was found that electronic cigarettes are a big trend, especially among young males. It can be seen that the use of electronic cigarettes is related to the use of conventional cigarettes and other drugs. Furthermore, many individuals believe that e-cigarettes are not harmful to health or are less harmful than conventional cigarettes. The awareness of the population about the risks of using electronic cigarettes becomes essential in the educational process, aiming to alert the harm that these electronic devices can cause. Therefore, given the scarcity of scientific publications on the use of electronic cigarettes in Brazil, it is suggested that more studies be carried out that address the issue under discussion.
\end{abstract}

Keywords: Electronic cigarette; Electronic device; Smoking.

\section{Resumen}

Los cigarrillos electrónicos se introdujeron en el mercado como una posible terapia para dejar de fumar. Sin embargo, los cigarrillos electrónicos pueden contener varias sustancias químicas, como la nicotina, además de metales pesados, como el hierro y el plomo. Este estudio tuvo como objetivo analizar el uso de cigarrillos electrónicos en Brasil, a través de una revisión integradora. Se utilizó una revisión integradora con búsqueda de datos realizada en las bases de datos Scielo, BVS y Academic Google, utilizando los siguientes descriptores: "uso de cigarrillos electrónicos" y "vapeo", realizada de septiembre a octubre de 2021. Se observó que existen pocos estudios realizados en Brasil sobre cigarrillos electrónicos. Se encontró que los cigarrillos electrónicos son una gran tendencia, especialmente entre los hombres jóvenes. Se puede observar que el uso de cigarrillos electrónicos está relacionado con el uso de cigarrillos convencionales y otras drogas. Además, muchas personas creen que los cigarrillos electrónicos no son dañinos para la salud o son menos dañinos que los cigarrillos convencionales. La conciencia de la población sobre los riesgos del uso 
de cigarrillos electrónicos se vuelve fundamental en el proceso educativo, con el objetivo de alertar sobre el daño que estos dispositivos electrónicos pueden causar. Por lo tanto, dada la escasez de publicaciones científicas sobre el uso de cigarrillos electrónicos en Brasil, se sugiere que se realicen más estudios que aborden el tema en discusión.

Palabras clave: Cigarrillo electrónico; Dispositivo electrónico; Fumar.

\section{Introdução}

O tabagismo se caracteriza pela dependência psicológica do tabaco, sendo a nicotina a responsável pela dependência química (Marato, 2014). Nesse aspecto, o tabagismo se configura como um grande problema de saúde pública na atualidade. É considerado fator de risco para o desenvolvimento de doenças não transmissíveis, como diabetes, doenças cardiovasculares, câncer e doenças respiratórias (Cavalcanti, 2018). Destarte, o tabaco mata cerca de 5 milhões de pessoas em todo o mundo, a cada ano (Focchi \& Braun, 2005).

Segundo a Organização Mundial da Saúde (OMS), aproximadamente 22,7\% da população mundial, acima de 15 anos, utiliza frequentemente cigarros (Leite et al., 2021). No Brasil, de acordo com o Vigitel 2020, o percentual de fumantes com 18 anos ou mais é de 9,5\% (Brasil, 2021).

No século XX, o tabagismo foi impulsionado, sobretudo pelas propagandas e pelo cinema. Nesse aspecto, no Brasil, a partir dos anos 70, as propagandas começaram a associar o ato de fumar com irreverência, ousadia e beleza (Cavalcanti, 2018). Entretanto, a prevalência do tabagismo no Brasil vem sendo reduzida nas últimas décadas. Desse modo, o controle do tabagismo vem sendo promovido desde 1989, por meio do Programa Nacional de Controle do Tabagismo (PNCT) (Silva, Martins, Faria \& Cotta, 2014).

Nesse contexto, o cigarro eletrônico foi introduzido no mercado como uma possível terapêutica na cessação do tabagismo (Barradas et al., 2021). Todavia, desde 2009, a Agência Nacional de Vigilância Sanitária (ANVISA) proibiu a comercialização, importação e propaganda de cigarros eletrônicos (Barufaldi et al., 2020).

Os cigarros eletrônicos são equipamentos que apresentam diferentes estilos e tamanhos (Almeida et al., 2020). Desse modo, esses cigarros são dispositivos de liberação de nicotina, movidos por bateria (Barufaldi et al., 2020). Com isso, o uso desses dispositivos está relacionado com o aumento no risco de alterações e infecções no trato respiratório. Ademais, o seu uso ocasiona aumento da contratilidade cardíaca e aumenta a pressão arterial (Batista et al., 2020).

Os fabricantes dos cigarros eletrônicos propagam que estes são mais seguros que os cigarros convencionais, utilizando o argumento que o eletrônico tem menos substâncias tóxicas do que o convencional (Scholz \& Abe, 2019). Ademais, por possuírem diversos aromas e sabores, muitos jovens são atraídos (Vargas et al., 2021). No entanto, os cigarros eletrônicos podem conter diversas substâncias químicas, como nicotina e aromatizantes, além de metais pesados, como ferro e chumbo (Pinto et al., 2020). Assim, o uso prolongado desses cigarros durante a adolescência pode contribuir para a iniciação do tabagismo (Knorst et al., 2014).

A presente pesquisa observou três aspectos acerca dos cigarros eletrônicos: a prevalência e perfil dos usuários que fazem o uso desses cigarros, a motivação para utilização e o conhecimento da população acerca dos cigarros eletrônicos. Portanto, diante do exposto, o presente estudo objetiva analisar o uso de cigarros eletrônicos no Brasil, por meio de uma revisão integrativa.

\section{Metodologia}

O presente estudo qualitativo descritivo, acerca do uso de cigarros eletrônicos, foi realizado no período de setembro a outubro de 2021, por meio de uma revisão integrativa de artigos da literatura científica. A pesquisa de artigos foi realizada nas bases eletrônicas Scientific Electronic Library Online (Scielo), Biblioteca Virtual em Saúde (BVS) e Google Acadêmico, por 
meio da leitura, análise, interpretação e seleção de artigos. A revisão integrativa sintetiza estudos disponíveis acerca de determinado tema (Souza et al., 2010). Nesse sentido, a revisão integrativa vem ganhando destaque nas pesquisas em saúde para auxiliar os estudos de revisão (Cecilio \& Oliveira, 2017).

Utilizou-se como estratégia de busca o operador Booleano OR e respectivos Descritores em Ciências da Saúde / Medical Subject Headings (DeCS/MeSH). Posto isso, foram utilizados os seguintes descritores: "Uso de cigarros eletrônicos" e "Vaping".

Foram selecionados artigos publicados nos últimos 10 anos, realizados no Brasil, apresentados em língua portuguesa, inglesa ou espanhola, que abordassem o uso de cigarros eletrônicos. Excluíram-se os artigos duplicados, revisões de literatura e os artigos não disponibilizados em acesso livre. Dessa forma, a Figura 1 faz o delineamento da seleção de artigos nas bases de dados.

Foram encontrados inicialmente 18 artigos na base de dados Scielo, 2.359 na BVS e 2.210 no Google Acadêmico, totalizando 4.587. Diante os critérios de inclusão e exclusão utilizados, restaram quatro para análise, sendo 3 da Scielo e 1 do Google Acadêmico.

Figura 1: Representação esquemática da seleção de artigos.

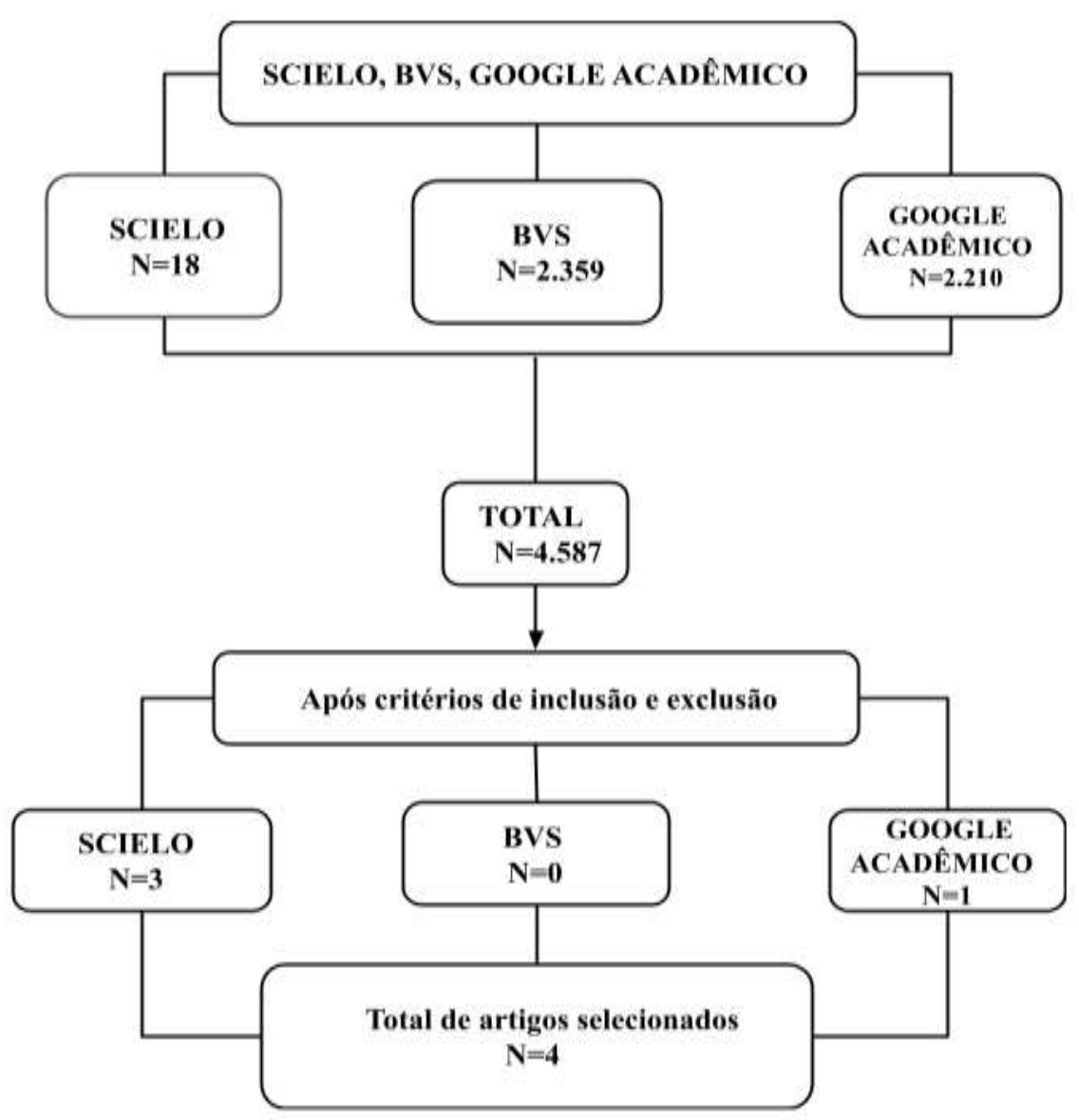

Fonte: Autores (2021).

\section{Resultados e Discussão}

A amostra final foi composta por quatro artigos. Nesse sentido, torna-se importante frisar que, foram incluídos apenas estudos realizados no Brasil, visto que a presente revisão integrativa teve como foco analisar a realidade brasileira, acerca do uso 
de cigarros eletrônicos. Portanto, apesar da temporalidade abarcar um período de 10 anos, observou-se que ainda são escassas as publicações científicas acerca da temática. A Tabela 1 apresenta informações dos artigos utilizados na presente pesquisa.

Tabela 1: Apresentação dos artigos incluídos na revisão integrativa.

\begin{tabular}{|c|c|c|c|}
\hline BASE DE DADOS & TITULO DO ARTIGO & AUTORES & $\begin{array}{c}\text { ANO DE } \\
\text { PUBLICAC̄ÃO }\end{array}$ \\
\hline Scielo & $\begin{array}{l}\text { Conbecimento e uso de cigarros } \\
\text { eletrónicos e percepção de risco no Brasil: } \\
\text { resultados de um pais com requisitos } \\
\text { regulatórios rígidos }\end{array}$ & $\begin{array}{l}\text { Tânia Maria Cavalcante; André Salem Szklo; } \\
\text { Cristina de Abreu Perez; James F. Thrasher, Moyses } \\
\text { Szklo; Janine Ouimet; Shannon Gravely; Geoffrey T. } \\
\text { Fong; Liz Maria de Almeida }\end{array}$ & 2017 \\
\hline Scielo & $\begin{array}{l}\text { Conhecimento e uso do cigarro eletrônico } \\
\text { entre estudantes da Universidade Federal } \\
\text { de Mato Grosso }\end{array}$ & $\begin{array}{l}\text { Wemerson José Concea de Oliveira; Alexandre } \\
\text { Figueiredo Zobiole; Claudia Bonadimen de Lima; } \\
\text { Rebeca Melo Zurita; Pedro Eduardo Muniz Flores; } \\
\text { Luis Guilherme Val Rodrigues; Raissa Carolina de } \\
\text { Assis Pinheiro; Victor Francisco Figueiredo Rocha } \\
\text { Soares e Silva }\end{array}$ & 2018 \\
\hline Google Acadêmico & $\begin{array}{l}\text { Nivel de conhecimento de alunos de } \\
\text { graduação em odontologia sobre cigarros } \\
\text { eletrônicos }\end{array}$ & $\begin{array}{l}\text { Eduarda Coelho Guckert; Caroline Zimmermann; } \\
\text { Maria Inés Meurer }\end{array}$ & 2021 \\
\hline Scielo & $\begin{array}{l}\text { Dispositivos eletrònicos para fumar nas } \\
\text { capitais brasileiras: prevalência, perfil de } \\
\text { uso e implicações para a Politica Nacional } \\
\text { de Controle do Tabaco }\end{array}$ & Neilane Bertoni; André Salém Szklo & 2021 \\
\hline
\end{tabular}

Fonte: Autores (2021).

Apesar da prevalência de uso de cigarros convencionais ter sido reduzida ao longo dos anos, os cigarros eletrônicos vêm ganhando grande espaço atualmente no Brasil. Nesse sentido, Bertoni e Szklo (2021) promoveram um estudo para estimar a prevalência de uso de dispositivos eletrônicos para fumar (DEFs). Desse modo, foi estimado que cerca de 6,7\% da população das capitais brasileiras acima de 18 anos já fez o uso de dispositivos eletrônicos para fumar, bem como 2,32\% façam uso ocasional ou diário destes dispositivos. Com isso, pode-se verificar que a prevalência não se mostra considerada alta. Todavia, evidencia-se a existência da preocupação de que possa haver um aumento no uso de dispositivos eletrônicos para fumar nos próximos anos.

Além disso, foi relatado maior prevalência de utilização de dispositivos eletrônicos para fumar (DEFs) entre os sujeitos que consomem álcool de forma abusiva. Dessa forma, pode-se observar que o consumo de álcool como expressivo fator contribuinte para o uso de DEFs, bem como o uso destes dispositivos favorecem o uso de álcool e outras drogas.

O estudo de Bertoni e Szklo (2021) ainda observou que boa parte dos indivíduos na faixa etária de 18 a 24 anos, atualmente fumantes, iniciaram ou mantiveram o uso de cigarros convencionais após o uso de cigarros eletrônicos. Desse modo, diante desse resultado, fica evidente que o consumo de cigarros eletrônicos se apresenta como porta de entrada para utilização de cigarros convencionais. Ademais, o estudo ainda mostrou que a prevalência de uso atual de DEF se apresenta maior entre o público masculino.

Em uma pesquisa realizada por Oliveira et al. (2018) com universitários, constatou-se que quanto mais jovem o estudante, maior chance de conhecer o cigarro eletrônico. Dessa forma, esse interesse em experimentar o cigarro eletrônico, pode ser justificado pelo fato da adolescência se apresentar como uma fase de descobertas, na qual o adolescente se torna atraído para 
experimentar e conhecer coisas novas. Assim, os adolescentes se tornam vulneráveis para fazer o uso de cigarros eletrônicos, mesmo conhecendo os riscos que estes podem ocasionar.

O estudo ainda observou existir relação com os indivíduos que experimentaram cigarro eletrônico com a presença de fumantes na família. Nesse aspecto, mostra-se possível de perceber que o âmbito familiar está ligado diretamente com o uso de cigarros eletrônicos. Destarte, se o indivíduo vê um familiar utilizando cigarros convencionais, isso pode estimulá-lo a também a utilizar, bem como a fazer o uso de cigarros eletrônicos.

O incentivo social, principalmente a influência de amigos, acaba contribuindo para a utilização. O estudo de Guckert et al. (2021) mostrou que cerca $24 \%$ dos participantes da pesquisa alegaram que se o cigarro eletrônico fosse oferecido por um amigo, eles provavelmente fariam o uso. Diante desse contexto, pode-se observar que diversos jovens utilizam cigarros eletrônicos, no intuito de ser aceito em determinado grupo social.

A percepção acerca dos riscos dos cigarros eletrônicos se mostra preocupante. Um estudo desenvolvido por Cavalcante et al. (2017) revelou que os fumantes que conheciam os cigarros eletrônicos, muitos acreditavam que estes eram menos nocivos em relação aos cigarros convencionais. Entretanto, os cigarros eletrônicos são tão prejudiciais à saúde quanto os cigarros tradicionais, pois possuem substâncias tóxicas, além de nicotina. Portanto, muitos indivíduos não têm conhecimento acerca dos riscos que os dispositivos eletrônicos para fumar podem ocasionar na sua saúde, por isso, fazem o uso.

Apesar de ser proibida a comercialização de cigarros eletrônicos no Brasil, a falta de fiscalização e o desrespeito à lei se torna muito evidente. Esse fato favorece para maior vulnerabilidade e exposição de indivíduos com interesse em adquirir CE. Nesse contexto, mesmo diante das restrições para o uso, o estudo desenvolvido por Cavalcante et al. (2021) estima que cerca de $6,86 \%$ da população das capitais brasileiras faça o uso dual de cigarros eletrônicos e cigarros convencionais.

\section{Considerações Finais}

Os cigarros eletrônicos se apresentam como grande tendência, sobretudo entre os jovens do sexo masculino. Entretanto, apesar de ser proibida a comercialização de cigarros eletrônicos no Brasil, diversas pessoas ainda fazem o uso atualmente. Assim, fica demonstrada a necessidade de intensificar a fiscalização, visando diminuir o acesso e uso desses dispositivos.

Pôde-se verificar que a utilização de cigarros eletrônicos tem relação com o uso de cigarros convencionais e outras drogas. Ademais, a influência de amigos e familiares contribui para utilização de cigarros eletrônicos.

Diversos indivíduos acreditam que os cigarros eletrônicos não fazem mal à saúde ou são menos nocivos que os cigarros convencionais. Destarte, a sensibilização da população acerca dos riscos do uso de cigarros eletrônicos se mostra imprescindível no processo educativo. Logo, o uso desse dispositivo eletrônico, enquanto veículo para uso de drogas, deve ser mais trabalhado nas escolas, visando alertar os seus malefícios e, por conseguinte, preservar a saúde e o bem-estar da população.

Portanto, diante da escassez de publicações científicas acerca do uso de cigarros eletrônicos no Brasil, sugere-se a realização de mais estudos. Espera-se ter contribuído para reflexão e alerta acerca desse desafio para saúde pública no Brasil.

\section{Referências}

Almeida, P. C. V., Silveira, M. B., Poiano, R., Américo, B., Padula, A. L., \& Santos, J. N. G. (2020). Lesões Pulmonares Associadas ao Uso do Cigarro Eletrônico. São Paulo: Blucher, 6(4), 92-120.

Bertoni, N., \& Szklo, A. S. (2021). Dispositivos eletrônicos para fumar nas capitais brasileiras: prevalência, perfil de uso e implicações para a Política Nacional de Controle do Tabaco. Cadernos de Saúde Pública, 37(7), e00261920-e00261920.

Barradas, A. D. S. M., Soares, T. O., Marinho, A. B., dos Santos, R. G. S., \& da Silva, L. I. A. (2021). Os riscos do uso do cigarro eletrônico entre os jovens. Global Clinical Research Journal, 1(1), 1-8. 
Barufaldi, L. A., Guerra, R. L., Albuquerque, R. C. R., Nascimento, A., Chança, R. D., Souza, M. C., \& Almeida, L. M. (2020). Risco de iniciação ao tabagismo com o uso de cigarros eletrônicos: revisão sistemática e meta-análise. Ciência \& Saúde Coletiva. http://www.cienciaesaudecoletiva.com.br/artigos/risco-deiniciacao-aotabagismocom-o-uso-de-cigarros-eletronicos-revisao-sistematica-e-metaanalise/17801?id=17801.

Batista, B. G., Batista, B. G., Lemos, L., de Carvalho, A. D. P. M., Maia, I. D. A. M., \& Tavares, A. G. O. R. (2020). Uso indiscriminado do cigarro eletrônico e seus malefícios ao trato respiratório. Atena Editora, 6(16), 145-152.

Brasil. (2020). Ministério da Saúde. Secretaria de vigilância em Saúde. Departamento de Análise em Saúde e Vigilância de Doenças Não Transmissíveis. Vigilância de fatores de risco e proteção para doenças crônicas por inquérito não telefônico: estimativas sobre frequência e distribuição sociodemográfica de fatores de risco e proteção para doenças crônicas nas capitais dos 26 estados brasileiros e no Distrito Federal em 2020. Brasília: Ministério da Saúde. https://www.gov.br/saude/pt-br/centrais-de-conteudo/publicacoes/publicacoes-svs/vigitel/relatorio-vigitel-2020-original.pdf.

Cavalcante, T. M., Szklo, A. S., Perez, C. D. A., Thrasher, J. F., Szklo, M., Ouimet, J., \& Almeida, L. M. D. (2017). Conhecimento e uso de cigarros eletrônicos e percepção de risco no Brasil: resultados de um país com requisitos regulatórios rígidos. Cadernos de Saúde Pública, 33 (supl.3): e00074416.

Cavalcanti, T. M. (2018). Cigarro eletrônico: representações sociais entre os seus consumidores [Tese Doutorado em Oncologia]. Instituto Nacional de Câncer José Alencar Gomes da Silva.

Cecilio, H., \& Oliveira, D. C. (2017). Modelos de revisão integrativa: discussão na pesquisa em Enfermagem. Ciaiq, 2, 764-772.

Focchi, G. R. A., \& Braun, I. M. (2005). Tratamento farmacológico do tabagismo. Archives of Clinical Psychiatry, $32(5), 259-266$.

Guckert, E. C.., Zimmermann, C., \& Meurer, M. I. (2021). Nível de conhecimento de alunos de graduação em odontologia sobre cigarros eletrônicos. Revista Abeno, 21(1), 1099-1099.

Knorst, M. M., Benedetto, I. G., Hoffmeister, M. C., \& Gazzana, M. B. (2014). Cigarro eletrônico: o novo cigarro do século 21?. Jornal Brasileiro de Pneumologia, 40, 564-572.

Leite, M. O., de Melo, L. A., Moraes, N. M. M. S., de Oliveira, F. A. F., \& de Oliveira, J. S. (2021). Cigarros eletrônicos: auxílio na cessação do tabagismo ou um novo risco à saúde? Caderno de Graduação-Ciências Biológicas e da Saúde, 6(3), 212-212.

Morato, C. S. (2014). O uso do cigarro e seus malefícios na Unidade de Saúde CAIC em Patos de Minas/MG [Monografia (Especialização em Atenção Básica em Saúde da Família), Universidade Federal do Triângulo Mineiro]. Nescon. https://www.nescon.medicina.ufmg.br/biblioteca/imagem/6172.pdf.

Oliveira, W. J. C. D, Zobiole, A. F., Lima, C. B. D., Zurita, R. M., Flores, P. E. M., Rodrigues, L. G. V., \& Silva, V. F. F. R. (2018). Conhecimento e uso do cigarro eletrônico entre estudantes da Universidade Federal de Mato Grosso. Jornal Brasileiro de Pneumologia, 44(5), 367-369.

Pinto, B. C. M., de Lima, M. M. B., Torres, G. G., Teixeira, I. D., Rodrigues, J. C., Pontelli, L. H. B. S., \& de Paiva, F. V. A. (2020). Cigarros eletrônicos: efeitos adversos conhecidos e seu papel na cessação do tabagismo. Revista Eletrônica Acervo Saúde, 12(10), e4376-e4376.

Scholz, J. R., \& Abe, T. O. (2019). Cigarro Eletrônico e Doenças Cardiovasculares. Revista Brasileira de Cancerologia, 65(3), 1-3.

Silva, S. T. D., Martins, M. C., Faria, F. R. D., \& Cotta, R. M. M. (2014). Combate ao Tabagismo no Brasil: a importância estratégica das ações governamentais. Ciência \& Saúde Coletiva, 19(02), 539-552.

Souza, M. T., Silva, M. D., Carvalho, R. D., Souza, M. D., Silva, M. D., \& Carvalho, R. D. (2010). Revisão integrativa: o que é e como fazer. Einstein (São Paulo), 8(1), 102-106

Vargas, L. S., Araújo, D. L. M., de Noronha, L. C., Carvalho, L. A. A., Mota, M. F. Q., Alvarenga, F. P., Campos, G. M. O., Lima, A. K. M., Oliveira, V. G., \& Barbosa, A. C. A. (2021). Riscos do uso alternativo do cigarro eletrônico: uma revisão narrativa. Revista Eletrônica Acervo Científico, 30 , e8135. 